\title{
A "chacra" e a diversidade vegetal na Morraria em Mato Grosso
}

\author{
The "chakra" and plant diversity in Morraria in Mato Grosso
}

\section{Le "chakra» et la diversité des plantes dans Morraria dans le Mato Grosso}

El "chakra" y la diversidad de plantas en Morraria en Mato Grosso

\author{
Sandro Nunes Vieira* \\ (sandrovieira@gmail.com) \\ Rodrigo Aleixo Brito de Azevedo** \\ (rodrigo_azevedo@unilab.edu.br) \\ Maria de Fatima Barbosa Coelho* \\ (coelhomfstrela@gmail.com) \\ Regiane Correia de Oliveira* \\ (regiane@gmail.com)
}

Recebido em 11/12/2015; revisado e aprovado em 28/01/2016, aceito em 15/04/2016

DOI: http:/ / dx.doi.org/10.20435/1984-042X-2016-v.17-n.3(04)

\begin{abstract}
Resumo: O objetivo deste trabalho foi descrever unidade de manejo "chacra" e a diversidade vegetal na região de Morraria em Mato Grosso. Foram utilizadas técnicas qualitativas de observação direta e observação participativa. Os tamanhos das "chacras" não excederam a 1,5 hectares. Os informantes identificaram 109 espécies vegetais. As "chacras" tem as funções de ofertar trabalho aos idosos, otimização da mão de obra e de recursos financeiros, alternativa de alimentação e de renda para as famílias e fonte de material genético para as roças.
\end{abstract}

Palavras-chave: agricultura tradicional; diversidade agrícola; Cáceres.

Abstract: The aim of this study was to describe management unit "chakra" and plant diversity in Morraria region in Mato Grosso. Qualitative techniques of direct observation and participant observation were used. The sizes of the "chakras" did not exceed 1.5 hectares. Informants identified 109 plant species. The "chakras," it has the functions of job offer for the elderly, optimization of labor and financial resources, alternative of food and income for families and genetic material source for the fields.

Key words: traditional agriculture; agricultural diversity; Cáceres.

Résumé: L'objectif de cette étude était de décrire l'unité de gestion «chakra» et la diversité des plantes dans la région Morraria dans le Mato Grosso. Les techniques qualitatives de l'observation directe et l'observation des participants ont été utilisés. Les tailles des "chakras" ne dépassent pas 1,5 hectares. Informateurs identifié 109 espèces végétales. Les «chakras», il a les fonctions de l’offre d'emploi pour les personnes âgées, l'optimisation de la main-d'œuvre et des ressources financières, de la nourriture et de revenus alternatifs pour les familles et source de matériel génétique pour les champs.

Mots-clés: agriculture traditionnelle; diversité agricole; Cáceres.

Resumen: El objetivo de este estudio fue describir la unidad de gestión "chakra" y la diversidad de plantas en la región Morraria en Mato Grosso. Se utilizaron técnicas cualitativas de observación directa y observación participante. Los tamaños de los “chakras" no excediera de 1,5 hectáreas. Los informantes identificaron 109 especies de plantas. Los "chakras", que tiene las funciones de oferta de empleo para las personas mayores, la optimización del trabajo y los recursos financieros, alternativa de alimentos e ingresos para las familias y materiales de base genética para los campos. Palabras clave: agricultura tradicional; diversidad agrícola; Cáceres

\footnotetext{
* Universidade Federal de Mato Grosso (UFMT), Cuiabá, Mato Grosso, Brasil.

** Universidade da Integração Internacional da Lusofonia Afro-Brasileira (UNILAB), Redenção, Ceará, Brasil.
} 


\section{INTRODUÇÃO}

Morraria é a denominação local utilizada para nomear uma extensa região no município de Cáceres, Estado de Mato Grosso, delineada por serras e vales sob vegetação de Cerrado, reconhecida nos estudos geomorfológicos como a unidade de relevo Província Serrana. A Morraria conforma um território para os morroquianos e é neste território que vem mantendo formas locais de construir seu próprio conhecimento, através da observação, classificação e experimentação.

Os agricultores da Morraria abasteciam com alguns gêneros alimentícios a sede do município até a década de 1960. $\mathrm{O}$ arroz, feijão e outros produtos, como a rapadura que, até hoje é fabricada artesanalmente em engenhos esculpidos em madeiras e movimentados por animais, eram transportados em carros de boi, para assim, serem comercializados no perímetro urbano.

Os trabalhos até agora realizados na região da Morraria identificaram e descreveram: a roça (GODOY, 2004; COSTA, 2004; MENDES, 2005; BASTOS, 2004), o quintal (GODOY, 2004; OLIVEIRA, 2006), o pasto (BASTOS, 2004), os recursos madeireiros (MENDES, 2005), as áreas de uso comum (BASTOS, 2004), os recursos naturais (RAMOS, 2006), os condicionantes de procedimentos técnicos (COSTA, 2004), a percepção e usos dos solos segundo os agricultores (ALVES, 2004).

Em pesquisa sobre os quintais da Morraria, Godoy (2004) teve acesso à nominação de "chacra", o que a levou a fazer uma pequena nota em seu trabalho. Pelas indicações de Godoy (2004), sabia-se que essa nominação era local e que essa unidade de manejo localizava-se nas proximidades da residência do agricultor, sendo muitas vezes associada com os quintais e as roças.
A "chacra" chama a atenção, por ser um desses elementos da multifacetada agricultura tradicional, aqui entendida como aquela que não está completamente inserida nos modelos da agricultura industrial (AZEVEDO, 2003), e, por ser a agricultura tradicional uma construção complexa de elementos diversos, é necessário um olhar mais aprofundado e cuidadoso nos elementos que a compõem. Portanto a abordagem em forma de sistemas é importante para a compreensão mais ampla de suas características e particularidades, descrevendo seus elementos e as relações que estes estabelecem entre si, tanto no tempo como no espaço (COSTA, 2004).

No entanto, para se entender um sistema, é necessário antes de qualquer ação saber identificá-lo, conhecendo seu conteúdo e estabelecendo seus limites, pois o relacionamento entre as partes e a resposta destas aos estímulos externos determina seus contornos. Por sua vez, Azevedo (2004) argumenta que o estudo do sistema se presta a uma descrição estrutural da realidade, devendo ser percebida a diferença entre a realidade e sua representação, pois é uma formulação teórica que objetiva a compreensão da realidade. Assim, para sistemas agrícolas, há três conceitos que permitem distinguir os problemas e as relações nas diferentes escalas que são: sistema agrário, sistemas de produção e sistemas de cultivo e criação (AZEVEDO, 2004).

O objetivo deste estudo foi descrever a estrutura e a diversidade vegetal da unidade de manejo "chacra" na morraria, Cáceres, Mato Grosso.

\section{MATERIAL E MÉTODOS}

O estudo foi conduzido na região da Morraria em Cáceres, Estado de Mato Grosso (Figura 1), no período de janeiro a dezembro de 2006. 

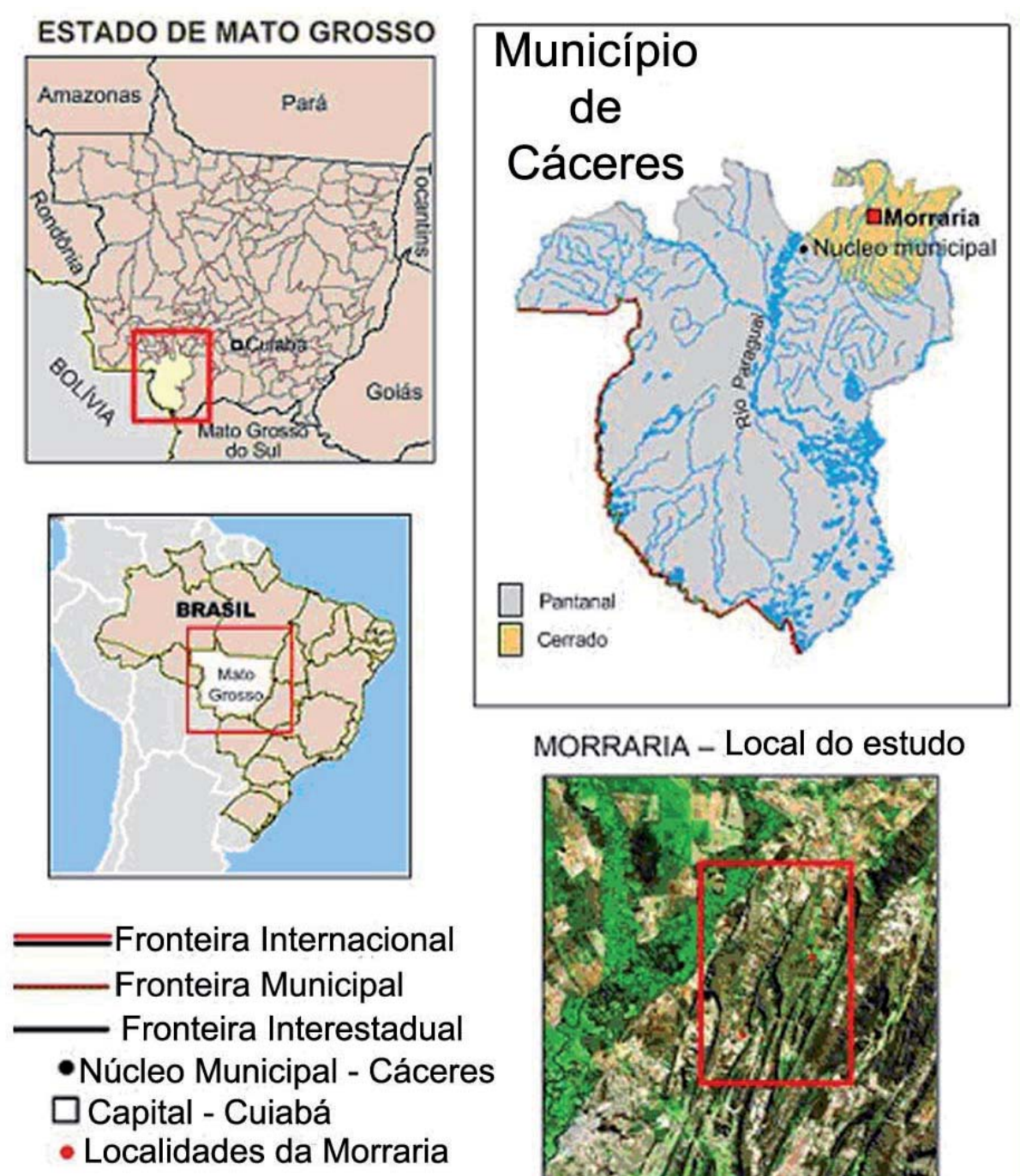

MORRARIA - Local do estudo

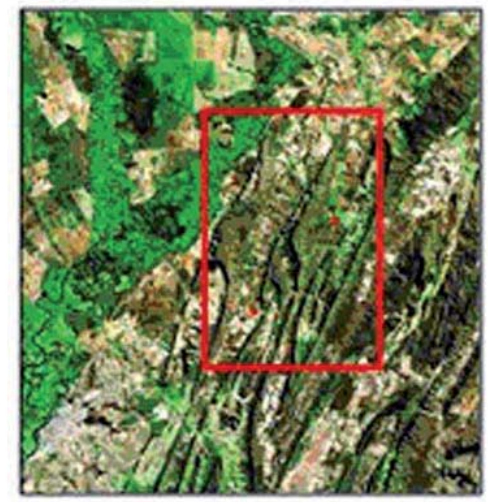

Figura 1 - Localização da Região da Morraria em Cáceres, Mato Grosso, Brasil

Fonte: Aguiar (2006).

A descrição da "chacra" segue a lógica de sistemas, por entender que essa unidade de manejo é e faz parte de um arranjo de componentes físicos unidos ou relacionados de tal maneira, que formam e atuam como uma unidade, entidade ou um todo (HART, 1985). As técnicas de pesquisa utilizadas foram: revisões bibliográficas; entrevistas a partir de um guia de campo; questionários; diário de campo; observação direta e observação participativa ou apontamento e consulta a técnicos e especialistas (MENDES, 2005; VIEIRA, 2003 AZEVEDO; COELHO 2002; VIERTLER, 2002; AZEVEDO, 2001; MONTEIRO;
MONTEIRO, 2000; COTTON, 1996; LAKATOS, 1985).

As informações foram obtidas junto a 24 informantes (Tabela 1), com níveis de informações diferentes, decorrentes, por vezes, da disponibilidade do informante, pouco conhecimento sobre o assunto ou ainda, informações somente sobre tratos culturais, o que, na fase estatística, culminou na ausência destes (por não terem informações que fossem contempladas na análise). Esses informantes estão distribuídos nas comunidades de Nossa Senhora do Carmo, Nossa Senhora da Guia e Sagrado Coração de Jesus, todas na região da Morraria. 
Tabela 1 - Características dos entrevistados em Morraria, Cáceres, Mato Grosso, 2007

\begin{tabular}{|c|c|c|c|c|c|}
\hline \multicolumn{2}{|c|}{$\begin{array}{l}\text { Sexo dos } \\
\text { informantes }\end{array}$} & \multirow[t]{2}{*}{ Comunidade } & \multirow[t]{2}{*}{ Localidade } & \multirow[t]{2}{*}{ Coordenadas } & \multirow[t]{2}{*}{ Localização } \\
\hline Masc. & Fem. & & & & \\
\hline três & quatro & $\begin{array}{l}\text { Nossa Senhora } \\
\text { do Carmo }\end{array}$ & Taquaral & $\begin{array}{l}15^{\circ} 58^{\prime} 56^{\prime \prime} \text { latitude } \\
\text { sul e } 57^{\circ} 31^{\prime} 14^{\prime \prime} \\
\text { longitude oeste de } \\
\text { Gr }\end{array}$ & $\begin{array}{l}\text { km } 22 \text { na rodovia MT- } \\
\text { 343, de Cáceres a Barra } \\
\text { dos Bugres }\end{array}$ \\
\hline quatro & -- & $\begin{array}{l}\text { Nossa Senhora } \\
\text { da Guia }\end{array}$ & Chapadinha & & $\begin{array}{l}\text { A comunidade está } \\
\text { localizada na bocaina do }\end{array}$ \\
\hline dois & -- & $\begin{array}{l}\text { Nossa Senhora } \\
\text { da Guia }\end{array}$ & $\begin{array}{l}\text { Barreiro } \\
\text { Vermelho e } \\
\text { Minador }\end{array}$ & $\begin{array}{l}15^{\circ} 54^{\prime} 40^{\prime \prime} \text { latitude } \\
\text { sul e } 57^{\circ} 31^{\prime} 03^{\prime \prime} \\
\text { longitude oeste de } \\
\text { Gr }\end{array}$ & $\begin{array}{l}\text { Taquaral, entre a serra } \\
\text { Taquaral e a serra do } \\
\text { Poção, do km } 24 \text { ao km } \\
30 \text { da rodovia MT- } 343\end{array}$ \\
\hline sete & uma & $\begin{array}{l}\text { Sagrado Coração } \\
\text { de Jesus }\end{array}$ & Santana & & $\begin{array}{l}\text { Aproximadamente } \\
20 \mathrm{~km} \text { da comunidade }\end{array}$ \\
\hline dois & uma & $\begin{array}{l}\text { Sagrado Coração } \\
\text { de Jesus }\end{array}$ & Anhumas & $\begin{array}{l}15^{\circ} 53^{\prime} 40^{\prime \prime} \text { latitude } \\
\text { sul e } 57^{\circ} 26^{\prime} 59^{\prime \prime} \\
\text { longitude oeste de } \\
\text { Gr }\end{array}$ & $\begin{array}{l}\text { de Nossa Senhora do } \\
\text { Carmo, distante da } \\
\text { rodovia MT-343 }\end{array}$ \\
\hline
\end{tabular}

\section{RESULTADOS E DISCUSSÃO}

Os agricultores diferenciam "chacra" de roça e quintal. Para o Sr. Cipriano é bom ter uma "chacra", pois "um dia que a gente precisa, tem serviço perto de casa pra fazer" e, assim, o "cara" tem que manter o local limpo, para ajudar a visualização de animais silvestres que vêm devorar os animais de "terreiro". Algumas diferenças são observadas pelo agricultor entre "chacra", a roça, o quintal e a chácara. Alguns desses exemplos ainda são relacionados aos aprendizados na infância, como o exemplo de que, quando o tio falava de ir a uma "chacra", ele já sabia que era uma unidade de manejo pequena, menor que uma roça, que era de um alqueire e meio.

Outra diferença entre a roça e a "chacra" é que "a chacra não muda de lugar, a roça muda, quando acaba com a mata, tem que mudar, para crescer de novo". Entre "chacra" e quintal, as diferenças se dão em relação ao espaço que o quintal ocupa, que em geral é o mesmo da residência; os cultivos por vezes são os mesmos (frutíferas, condimentares, etc), mas, nas "chacras", diferente do quintal, quase não há plantas ornamentais e, ainda, em boa parte dos quintais, no fundo há cerca para restringir a entrada de animais de grande porte. A "chacra" é uma rocinha pequena com tamanhos entre 0,43 a 1,21 ha, com produção voltada ao consumo próprio e das criações, e com eventual comercialização da farinha, que é produzida com mandioca da "chacra".

Segundo o informante, os produtos da roça é que são comercializados. $\mathrm{Na}$ "chacra [...] planta o que quer, mandioca, cará, cana, [...] é sortido de tudo [...]" e, dentro desse sortimento, há espécies comuns à roça e ao quintal, como quiabo, melancia, laranja, jabuticaba, goiaba, gergelim, coco da Bahia, cará, batata doce e abóbora, além de algumas nativas como barbatimão, paratudo, angelim, etc. Assim, a "chacra" possui, além da produção de alimentos para a subsistência, a função de produção de mudas e sementes que serão destinadas à roça.

O principal cultivo designa o nome da unidade de manejo do Sr. Cipriano: "Chacra de Mandioca", estrategicamente construída em uma antiga área de capoeirão, deixada pelo proprietário anterior e com a possibilidade de se manter por 
até quatro anos, sem uso de adubo, como informado pelo Sr. Cipriano. Essa unidade de manejo com 0,3 ha está a 15 metros de distância da residência, o que facilita a lida e o acesso aos mantimentos e, pelo tamanho reduzido, colabora para a não contratação da mão de obra externa. Depois da "separação do taquaral, as pessoas quase não se ajudam na demão (mutirão), ... só se preocupam durante os muxiruns (quando há) em beber e comer, antes, não era assim".

$O$ período em que em geral se começa a feitura da "chacra" é no início da época de chuva, em que, entre setembro e outubro, se limpa a capoeira e, até junho, o plantio é efetuado. Mas, devido às mudanças climáticas, muitos cultivos tiveram alterações em seu ciclo de plantio, exemplo dado da bananeira e arroz, que atualmente é em outubro, mas antigamente era em setembro.

Outro agricultor entrevistado, o Sr. Catulino, afirma que "alguns agricultores não usam essa nominação de chacra, pois, não quer usar o jeito de nós falar, nossa descendência [...] jeito de pai, de avós, daí ele vai modificando o jeito de falar [...] deveria falar, a gente já pegou esse idioma assim [...] é um conhecimento de família". A "chacra" que o informante possui tem cerca de 0,5 ha, é menor que a roça, 2,5 a 7,5 ha, "assim, devido a esse tamanho, as atividades que se têm na roça demandam contratação de mão de obra externa". Outra diferença entre roça e "chacra" se dá na necessidade de avanços ou mudanças em seu território (da roça) para buscar solo com qualidade melhor para a produção. Diferente da "chacra", pois devido ao número menor de indivíduos cultivados, a área ocupada e o impacto são menores, assim, o local pode ser utilizado por muito tempo e, quando observada a invasão de ervas daninhas ou enfraquecimento do solo, o local pode ser deixado em pousio para um posterior retorno.

Espécies como milho, feijão, arroz, mamão, mandioca e banana coexistem nas duas unidades de manejo e, apesar das particularidades de cultivos e tamanhos, se podem fazer juntas, em uma mesma propriedade, a "chacra" e a roça. Das diferenças e similaridades entre "chacras" e quintais, se tem que o quintal possui tamanho menor que a "chacra"; em ambas há as cercas de três fios de arame farpado; os cultivos no quintal são em menor quantidade de indivíduos, mas com maior diversidade de espécies, tais como: as plantas ornamentais, medicinais, frutíferas, hortícolas e condimentares, sendo muitas dessas comuns às duas unidades de manejo, em um lugar separado do quintal, mas não muito distante dele.

Entre a "chacra" e a chácara, se tem as seguintes diferenças, conforme relatos dos agricultores: a localização da chácara é nos arredores ou mesmo no perímetro urbano. Seu tamanho é de 0,5 a 2 hectares e possui a produção voltada para comercialização, o que é raro na "chacra". Outra particularidade apontada pelo Sr. Catulino é que na produção, mesmo que com intuito comercial, a chácara, em muitos casos, tem a sua produção menor que na "chacra". Nesse caso, o fato da "imobilidade" da chácara acarreta o alagamento do solo, que afeta a produção durante o período de chuva, e ainda, por ser sempre em um mesmo local, o desgaste ou mesmo a terra com qualidade baixa é outro agravante.

Na Figura 2, observam-se os diferentes espaços reconhecidos pelos agricultores, incluindo a chacra. 


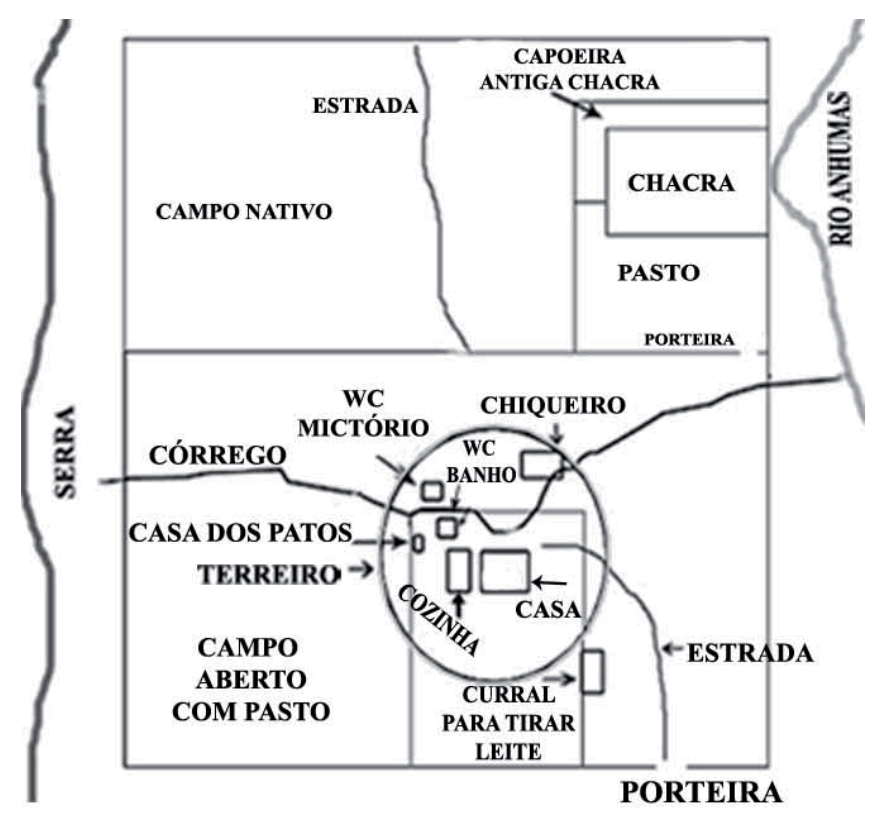

Figura 2 - Croqui de uma Chacra confeccionado junto ao agricultor

A "chacra" é caracterizada pelo senhor Catulino, com mais de 20 anos de experiência nesse assunto, como sendo uma roça pequena (consorciada) de 1 a 2 ha, de grande importância devido à produção para autoconsumo (e ainda para os animais e cessão dos excedentes aos vizinhos); facilidade ao acesso, devido à proximidade da residência e ainda, produzir semente para a outra roça, no período em que não pôde efetivar.

Nessa unidade de manejo, há diversidade de cultivos de ciclo curto e longo, em que existem variedades locais ou não, que também são comuns aos quintais, roças e ares de capoeira, como: o milho de três meses (para fazer curau) e o asteca; arroz de três e quatro meses; mandioca "liberata"; batata doce, cana, mamão, cara, abacaxi, abóbora, feijão, banana "salta veaco", rúcula, couve, alface, pimentão, maxixe, quiabo, pepino, abóbora, melancia, pimenta doce e pimenta malagueta.

No caso da atual "chacra", a distância entre ela e a casa está em aproximadamente 200 metros, e seu tamanho é de 0,5 alqueire (1,25 ha), que é outra característica ressaltada (tamanho menor que a roça e maior que o quintal).
O processo de derrubada e queima tem início no mês de maio, e o plantio, em outubro ou novembro, no começo de chuva. O casal que exerce as atividades na chacra, onde trabalham entre 7 a 12 dias por mês, e $5 \mathrm{~h}$ por dia, das $8 \mathrm{~h}$ às $11 \mathrm{~h}$ e das 14h às 16h (depende do sol ou da chuva). Por vezes, há a troca de dia (ajuda mútua ou de mão): “às vezes os cunhados Juscelino ou Paulo dá uma de mão do dia e não cobra nada" disse o Senhor Catulino.

A escolha do local se dá pela observação de características que o agricultor percebe como de "terra de produção", ou seja, terra preta, e que consiga manter a umidade; terras altas e que não alaguem facilmente e local de fácil lida, geralmente em capoeiras, que é uma vegetação boa, com pouca "galhada", diâmetro pequeno que facilita a derrubada e, por conseqüência, menor esforço do agricultor, que, no caso do Senhor Catulino, possui problemas de saúde.

Após 2 ou 3 anos de utilização do local, vários motivos contribuem para que essa área seja abandonada ou deixada em repouso (pousio). Entre eles se tem a possibilidade de implementar outra área de cultivo, pois há mais espaço; o aumento 
de ervas daninhas (sementeira); permitir a recuperação das qualidades do solo através dos processos naturais, e ainda, o local pode ser reutilizado para pastagem (capim Brachiaria).

Após um certo tempo de uso da terra, há ocorrência de plantas invasoras, como o carrapicho de árvore, picão, capim amargoso, capim mais fino e o capim bravo. Também aparece a lagarta "medi-palmo", que ataca as folhas do milho e feijão, e o "coró", que ataca os cultivos de mandioca e banana, provocando apodrecimento. Para tanto, o uso do inseticida fosforado, de nome comercial Folidol, se dá, como dito pelo informante: "depende do agitamento (quantidade) do inseto".

Apesar das pragas e plantas invasoras, o agricultor diz que a produção não é tão acentuada, em razão do ataque de animais silvestres, como o tatu, macaco, cutia, quati, mutum, veado etc., que se alimentam das plantas da chacra.

Segundo D. Antonia, a "chacra" se parece com um quintal com vegetais característicos da roça e do quintal. A informante complementa:

O quintal não sendo grande é chacrinha, até um alqueire [...] falo assim, porque meu pai fazia essa explicação [...] ali na Margarida, Celestino e Andresa, é quintal [...] mesmo que se plantar arroz, feijão e outros, ainda, não é Chacra, porque dá pouco, um pé aqui, outro ali $[\ldots]$, pois, a distância é pouca, três braças [...] a Chacra é mais espaciosa que o quintal [...] não é muito pregadinho da casa, tem que ser, um pouco longinho e espaciosa.

Nas Chacras, há espécies vegetais nativas, exóticas, madeireiras, medicinais, condimentares, aromáticas, alimentícias e ornamentais, com uso em construção, utensílios, lenha, estaca para cerca e sombra, entre outros usos. Foram citadas 109 etnoespécies, que são apresentadas nas Tabelas 2 e 3.

Algumas espécies nativas que foram observadas durante as visitas guiadas, ou citadas pelos agricultores, corroboram com o trabalho de Mendes (2005). A maioria dessas espécies apresenta diversos usos, com predominância de etnoespécies para construção. Possivelmente isso se deve à distancia e precariedade das estradas para Cáceres, e ao hábito cultural dos moradores de fazer casas de taipa. Apesar de representadas em menor número em relação às plantas exóticas, o cultivo das espécies nativas constitui importante banco de germoplasma, que garante a variabilidade genética de muitas dessas espécies (BRITO; COELHO, 2000).

Tabela 2 - Espécies nativas encontradas nas Chacras de Morraria, Cáceres, Mato Grosso. 2007

\begin{tabular}{|c|c|c|c|}
\hline Etnoespécies & Nome Científico & Família & Usos \\
\hline Acuri & Attalea phalerata (Mart.) Burret & Arecaceae & AL; CT;EC \\
\hline Amoreira & Maclura tinctoria & Moraceae & SB. \\
\hline Angelim & Vatairea macrocarpa (Benth.) Ducke & Fabaceae & LE; MD \\
\hline Barbatimão & Stryphonodendron adstringens(Mart.) & Mimosoideae & CT; ET;LE; MD \\
\hline Caiá & Spondia lutea L & Anacardiaceae & EC; LE;AL \\
\hline Cedro & Cedrela fissilis Vell. & Meliaceae & $\mathrm{CT}$ \\
\hline Cumbaru & Dipteryx alata Vog. & Fabaceae & CT; ET;SB; AL \\
\hline Fruta banana & Ecclinusa sp. & Sapotaceae & AL \\
\hline Jatobá & Hymenea stigonocarpa Mart. Ex Hayne & Caesalpiniaceae & CT; ET;LE; MD \\
\hline Jenipapo & Genipa americana L. & Rubiaceae & CT; ET;LE; CF;AL \\
\hline Louro-preto & Cordia glabrata (Mart).DC & Boraginiaceae & CT; ET; LE \\
\hline Mamica-de-porca & Zantoxylum rhoifolium Lam. & Rutaceae & ET; LE \\
\hline Mangava brava & Lafoensia pacari A St. - Hil. & Lythraceae & AL; MD;ET; CT;LE \\
\hline Marmelada & Alibertia edulis) (Vell.) K. Schum & Rubiaceae & AL; LE;MD \\
\hline Paratudo & Tabebuia aurea Manso & Bignoniaceae & $\mathrm{CT} ; \mathrm{MD} ; \mathrm{LE}$ \\
\hline Peroba-rosa & Aspidosperma sp & Apocynaceae & CT; CF;ET; LE \\
\hline
\end{tabular}

Legenda: AL: Alimento; CT: Construção; CF: Cabo de ferramenta; LE: Lenha; ET: Estaca para cerca; EC: Espera de caça; SB: Sombra; MD: Medicinal e UT: Utensílio. 
As etnoespécies introduzidas são muito diversificadas e usadas principalmente para alimentação $(68,7 \%)$ (Tabela
3). Esse fato proporciona à comunidade certa segurança alimentar em relação aos habitantes da cidade de Cáceres.

Tabela 3 - Etnoespécies cultivadas introduzidas na Morraria, Cáceres, Mato Grosso. 2007

\begin{tabular}{|c|c|c|c|}
\hline $\begin{array}{l}\text { Etnoespécies } \\
\end{array}$ & Nome Científico & Família & Usos \\
\hline Abacate & Persea americana Mill & Lauraceae & $\mathrm{AL}$ \\
\hline Abacaxi Perola & Ananas comosus (L.) Merril & Bromeliaceae & AL \\
\hline Abóbora & Cucurbita pepo L. & Cucurbitaceae & AL \\
\hline Acerola & Malpighia glabra L. & Malpighiaceae & $\mathrm{AL}$ \\
\hline Aipim & Manihot esculenta Crantz & Euphorbiaceae & AL \\
\hline Alface & Latuca sativa L. & Asteraceae & AL \\
\hline Algodão & Gossipium barbadense L. & Malvaceae & MD \\
\hline Amendoim & Arachis hipogaea L. & Fabaceae & AL \\
\hline Arroz agulhinha 3 meses & Oryza sativa L. & Poaceae & AL \\
\hline Arroz agulhinha 4 meses & Oryza sativa $\mathrm{L}$. & Poaceae & AL \\
\hline Arruda & Ruta graveolens L. & Rutaceae & MD \\
\hline Babosa & Aloe vera $\mathrm{L}$. & Liliaceae & MD \\
\hline Banana cabutá & Musa paradisíaca L. & Musaceae & $\mathrm{AL}$ \\
\hline Banana de fritar & Musa paradisíaca L. & Musaceae & $\mathrm{AL}$ \\
\hline Banana nanica & Musa paradisíaca L. & Musaceae & AL \\
\hline Batata Doce & Ipomoea batatas (L.) Lam. & Convolvulaceae & AL \\
\hline Beladona & Atropa belladona L.' & Solanaceae & MD \\
\hline Boldo & Plectranthus barbatus Andrews & Lamiaceae & MD \\
\hline Boldo do Chile & Peumus boldus Molina & Lamiaceae & MD \\
\hline Bucha & Luffa cylindrica (L.) Roem & Cucurbitaceae & $\mathrm{AL}$ \\
\hline Cabaça de árvore & Crescentia cujete L. & Bignoniaceae & UT \\
\hline Café & Coffea arabica $\mathrm{L}$. & Rubiaceae & AL \\
\hline Caju & Anacardium occidentale L. & Anacardiaceae & $\mathrm{AL}$ \\
\hline Cana de açúcar "Maria Pelada" & Saccharum officinarum $\mathrm{L}$ & Poaceae & $\mathrm{AL}$ \\
\hline Capim cidreira & Cybopogon citratus (DC.) Stapf. & Poaceae & MD \\
\hline Cará & Dioscorea alata L. & Dioscoreaceae & AL \\
\hline Cebola & Allium сера L. & Liliaceae & $\mathrm{AL}$ \\
\hline Cebolinha & Allium fistulosum L. & Liliaceae & AL \\
\hline Cenoura & Daucus carota L. & Apiaceae & AL \\
\hline Coco da Bahia & Cocos nucifera L. & Arecaceae & AL \\
\hline Colônia & Alpinia speciosa Schum & Zingiberaceae & MD \\
\hline Couve & Brassica oleraceae L. & Brasicaceae & AL \\
\hline Croá & Sicana odorífera Naudin & Cucurbitaceae & MD \\
\hline Fava branquinha & Vicia faba L. & Fabaceae & AL \\
\hline Fava rosinha & Vicia faba L. & Fabaceae & AL \\
\hline Feijão carioca & Phaseolus vulgaris L. & Fabaceae & AL \\
\hline Feijão de corda & Vigna unguiculata (L.) Walp & Fabaceae & AL \\
\hline Feijão guandu & Cajanus cajan L & Fabaceae & AL \\
\hline Feijão pastel & Vigna unguiculata (L.) Walp & Fabaceae & AL \\
\hline Feijão rosinha & Phaseolus vulgaris L. & Fabaceae & AL \\
\hline Gengibre & Zingiber officinale Roscoe & Zingiberaceae & MD \\
\hline Gergelim & Sesamum indicum L. & Pedaliaceae & AL \\
\hline Goiaba & Psidium guajava L & Myrtaceae & AL \\
\hline Graviola & Anona muricata L. & Annonaceae & AL \\
\hline Guariroba & Syagrus oleracea (Mart.) j Becc & Palmae & AL \\
\hline Guiné & Petiveria alliaceae $\mathrm{L}$. & Phytolaccaceae & MD \\
\hline Inhame & Dioscorea alata L. & Dioscoreaceae & $\mathrm{AL}$ \\
\hline Jabuticaba & Myrtus jaboticaba L. & Rutaceae & AL \\
\hline Jaca & Artocarpus heterophyllus Lam. & Moraceae & AL \\
\hline Laranja comum & Citrus aurantium L. & Rutaceae & AL \\
\hline Laranja misteriosa & Citrus aurantium L. & Rutaceae & AL \\
\hline Limão galego & Citrus limonia Osbeck & Rutaceae & AL \\
\hline Limão rosa & Citrus limonia Osbeck & Rutaceae & AL \\
\hline Limão Taiti & Citrus aurantifolia (Christm.) Swingle & Rutaceae & AL \\
\hline Mamão Castelo & Carica papaya L. & Caricaceae & AL \\
\hline Mamão Papaia & Carica papaya L. & Caricaceae & AL \\
\hline Mamona & Ricinus communis $\mathrm{L}$ & Euphorbiaceae & MD \\
\hline
\end{tabular}




\begin{tabular}{|c|c|c|c|}
\hline Etnoespécies & Nome Científico & Família & Usos \\
\hline Mandioca Liberata & Manihot esculenta Crantz & Euphorbiaceae & $\mathrm{AL}$ \\
\hline Mandioca amargozinha & Manihot esculenta Crantz & Euphorbiaceae & AL \\
\hline Mandioca vermelha & Manihot esculenta Crantz & Euphorbiaceae & $\mathrm{AL}$ \\
\hline Manga Bourbon & Mangifera indica L. & Anarcadiaceae & $\mathrm{AL}$ \\
\hline Mandioca branca & Manihot esculenta Crantz & Euphorbiaceae & AL \\
\hline Manga comum & Mangifera indica L. & Anacardiaceae & $\mathrm{AL}$ \\
\hline Macelinha & Achyrocline satureioides L. & Asteraceae & MD \\
\hline Maxixe & Cucumis anguria L. & Cucurbitaceae & AL \\
\hline Melancia & Citrullus lanatus L. & Cucurbitaceae & $\mathrm{AL}$ \\
\hline Mexerica & Citrus reticulata Blanco & Rutaceae & $\mathrm{AL}$ \\
\hline Milho Asteca & Zea maiz L. & Poaceae & AL \\
\hline Milho caiana & Zea maiz L. & Poaceae & AL \\
\hline Milho de 3 meses & Zea maiz L. & Poaceae & $\mathrm{AL}$ \\
\hline Milho de palha roxa & Zea maiz L. & Poaceae & AL \\
\hline Milho híbrido & Zea maiz L. & Poaceae & AL \\
\hline Moranga & Cucurbita pepo 1. & Cucurbitaceae & $\mathrm{AL}$ \\
\hline Nove horas & Portula grandiflora L. & Portulaceae & AL \\
\hline Palma & Opuntia ficus indica Mill & Cactaceae & MD \\
\hline Pepino & Cucumis satious L. & Cucurbitaceae & AL \\
\hline Pimenta do reino & Piper nigrum L. & Piperaceaea & AL \\
\hline Pimenta doce & Capsicum annum L. & Solanaceae & $\mathrm{AL}$ \\
\hline Pimenta Lambari & Capsicum baccatum L & Solanaceae & AL \\
\hline Pimenta malagueta & Capsicum frutescens L. & Solanaceae & AL \\
\hline Pimentão & Capsicum annuum L. & Solanaceae & $\mathrm{AL}$ \\
\hline Pinha & Annona squamosa L. & Annonaceae & AL \\
\hline Pitanga & Eugenia uniflora L. & Malpighiaceae & AL \\
\hline Quiabo & Abelmoschus esculentus (L.) Moench & Malvaceae & $\mathrm{AL}$ \\
\hline Quiabo de Angola & Hibiscus sabdarifa L. & Malvaceae & $\mathrm{AL}$ \\
\hline Romã & Punica granatum L. & Punicaceae & $\mathrm{MD} ; \mathrm{AL}$ \\
\hline Rúcula & Eruca sativa (Mill) Thell & Brasicaceae & AL \\
\hline Siriguela & Spondias purpurea L. & Anarcadiaceae & AL \\
\hline Tamarindo & Tamarindus indica L. & Caesalpinaceae & AL \\
\hline Tangerina & Citrus reticulata $\mathrm{L}$. & Rutaceae & $\mathrm{AL}$ \\
\hline Teca & Tectona grandis L. & Verbenaceae & ET \\
\hline Terramicina & Alternanthera brasiliana (L.) O Kuntze & Amaranthaceae & MD \\
\hline Urucum nativo & Bixa orellana L. & Bixaceae & $\mathrm{AL}$ \\
\hline
\end{tabular}

Legenda: AL: Alimento; ET: Estaca para cerca; MD: Medicinal e UT: Utensílio.

Na Amazônia, nos grupos de espécies botânicas utilizadas por populações sedentárias na forma de quintais caseiros, o de maior expressão, ou pelo menos o de maior visibilidade, é o da categoria "perene" (CLEMENT, 1999). Nesse grupo se encontram as árvores frutíferas comestíveis, que incorporam à alimentação diferentes fontes de vitaminas, de suma importância ao metabolismo fisiológico humano, podendo também oferecer sombra e lazer, além de se enquadrarem no grupo das medicinais alternativas (MADALENO, 2000).

Em Boa Vista, Roraima, Semedo e Barbosa (2007) verificaram que o cultivo de árvores frutíferas em quintais caseiros segue um padrão que concentra a escolha em poucas espécies, não-originárias da
Amazônia, mas tradicionalmente consagradas por seu êxito na produção de frutos. Nos quintais de Santarem, Pará, Winklerprins (2002) constatou a presença de 33 espécies de frutíferas em um total de 98 espécies.

A diversificação produz melhores efeitos nutricionais do que o cultivo de uma grande quantidade de uma ou duas espécies, e, nesse sentido, a maioria das frutas cultivadas nas chacras da Morraria é rica em vitamina $C$ (Tabela 4 ), tais como acerola, caju, goiaba e diversos citros. Entretanto o conjunto das frutíferas observadas é pobre em vitamina A (< 120 mg.100g-1), exceto manga e goiaba. Essa mesma observação é feita para proteínas (adequada > 2,5 g.100g-1), 
exceto para coco e jaca. O cultivo de coco (rico em proteínas) e o de manga (vitamina A), além de acerola, caju e goiaba (vitamina $\mathrm{C}$ ) indicam que a conjugação desses cultivos caseiros pode ser par- te de uma estratégia de sobrevivência (MADALENO, 2000), contribuindo para a suplementação alimentar de populações de baixa renda, como preconizado pela FAO (2004).

Tabela 4 - Estimativa da concentração de vitaminas e proteínas em algumas frutíferas das chacras de Morraria, Cáceres, Dados de IBGE (1981)

\begin{tabular}{lcrrrrrr}
\hline Nome científico/comum & $\begin{array}{c}\text { Proteína } \\
\text { g.100g-1 }\end{array}$ & $\begin{array}{c}\text { Vit A } \\
\text { Hg Eq. } \\
\text { Retinol }\end{array}$ & $\begin{array}{c}\text { Vit B1 } \\
\text { mg. 100g-1 }\end{array}$ & $\begin{array}{c}\text { Vit B2 } \\
\text { mg.100g-1 }\end{array}$ & $\begin{array}{c}\text { Vit C } \\
\text { mg.100g-1 }\end{array}$ & $\begin{array}{c}\text { \% Não- } \\
\text { comes- } \\
\text { tível }\end{array}$ \\
\hline Artocarpus integrifolia L. jaca & 2,7 & 39 & 0,09 & 0,11 & 9 & 55,0 \\
Anacardium occidentale L. cajueiro & 0,8 & 40 & 0,03 & 0,03 & 219 & 19,5 \\
Annona squamosa L. Fruta do conde & 1,6 & - & 0,11 & 0,15 & 35 & 42,5 \\
Carica papaya L. mamão & 0,5 & 37 & 0,03 & 0,03 & 46 & 29,5 \\
Citrus aurantium Osbeck laranja & 0,8 & 13 & 0,09 & 0,09 & 59 & 35,8 \\
Citrus aurantifolia Swingle limeira & 0,6 & 2 & 0,06 & 0,06 & 32 & 35,2 \\
Citrus limonia Osbeck limão & 0,6 & 2 & 0,06 & 0,02 & 51 & 54,5 \\
Cocos nucifera L. coco & 3,5 & - & 0,04 & 0,03 & 4 & 44,0 \\
Malpighia glabra L. acerola & 0,4 & - & - & - & 1678 & - \\
Mangifera indica L. manga & 0,5 & 210 & 0,05 & 0,06 & 53 & 39,7 \\
Musa paradisiaca L. banana & 1,5 & 38 & 0,05 & 0,06 & 17 & 29,7 \\
Psidium guajava L. goiaba & $\mathbf{x}$ & 174 & 143 & 7,4 & 91 & 63 \\
\hline
\end{tabular}

A unidade de produção "chacra" está inserida dentro do conceito de agricultura familiar no Brasil e, como tal, representa uma alternativa modeladora de um desenvolvimento menos excludente e ambientalmente mais equilibrado (SANTOS; MITJA, 2012).

O tema do desenvolvimento local vem sendo, nos últimos anos, objeto de intensos debates entre especialistas. Uma definição aceita para esse conceito é aquela, segundo a qual, o desenvolvimento local representa uma estratégia que deve assegurar para o território em questão - seja comunidade, município ou microrregião - uma melhoria das condições socioeconômicas, a médio e longo prazo (TABOSA et al., 2012). Para alguns especialistas, o termo "local" se insere no novo discurso das agências de desenvolvimento, públicas e privadas, com objetivo de captar recursos financeiros, enquanto, para outros, representa uma eficaz alternativa de melhoramento das condições de vida das comunidades, através de processos que buscam dotá-las de maior capacidade de gestão e de administração e seus próprios recursos e potencialidades.

Nas propriedades dos agricultores de Morraria, as chacras são responsáveis pela diversidade vegetal assegurando alimentos de qualidade para as famílias, empregam a mão de obra local que estaria afastada do processo agrícola em outros sistemas, como as pessoas mais velhas, auxilia a fixação do homem no campo e são manejadas de forma sustentável com os próprios recursos dos agricultores, além disso, ainda podem fornecer renda extra para as famílias. Em razão da distância de centros urbanos e da precariedade dos acessos às propriedades dos agricultores de Morraria, a manutenção das "chacras" contribui efetivamente para o desenvolvimento local. 


\section{CONSIDERAÇÕES FINAIS}

A unidade de manejo chacra representa para os marroquianos um reforço para o desenvolvimento local integrado às demais unidades de manejo, com a ênfase na fixação do homem ao campo por ser uma alternativa de cultivo para as pessoas idosas e enfermas, otimização da mão de obra e recursos financeiros, fonte alimentar e de renda para as famílias. Nos estudos com agricultores tradicionais, é necessário conhecer os termos e significados locais para entender os sistemas agrícolas, entretanto poucos estudos tratam desse tema.

\section{REFERÊNCIAS}

AGUIAR, M. V. de A. El aporte del conocimento local para el desarrolo rural: un estúdio de caso sobre el uso de La biodiversidad em dos comunidades campesinas tradicionales del Estado de Mato Grosso-Brasil. 2006. 744f. Tese (Doutorado em Agroecologia, Campesinato e História) - Instituto de Sociologia e Estudos Campesinos, Universidade de Córdoba, Córdoba, Espanha, 2006.

ALVES, H.S. Percepção dos agricultores em relação ao solo e seu uso, em comunidades tradicionais, da região da Morraria, Cáceres, MT. 2004. 390f. Dissertação (Mestrado em Agricultura Tropical) - Faculdade de Agronomia e Medicina Veterinária, Universidade Federal de Mato Grosso, Cuiabá, MT, 2004.

AZEVEDO, R. A. B. Descrição dos sistemas agrários de Mato Grosso - bases teóricas e critérios para descrição e análise. Cuiabá, MT, 2004. 27p. Texto produzido para disciplina de sistemas Agrícolas Tropicais.

AZEVEDO, R.A. B de.; COELHO M. F. B. Métodos de investigação do conhecimento popular sobre plantas medicinais. In: RODRIGUES, A. G. et al. Plantas medicinais e aromáticas: etnoecologia e etnofarmacologia. Viçosa: UFV, 2002.

AZEVEDO, R. A. B. de. Indicadores agronômicos em unidades de produção de agricultura familiar. 2001. 306f. Tese (Doutorado em Fitotecnia) Departamento de Fitotecnia, Universidade Federal de Viçosa, Viçosa, MG, 2001.
AZEVEDO, R. A. B. de. Os agricultores tradicionais e a agronomia: a difícil compatibilidade dos modelos conceituais. In: COELHO, M. F. B.; COSTA Jr., P.; DOMBROSKI, J. L. D. Diversos olhares em etnobiologia, etnoecologia e plantas medicinais. Cuiabá, MT: Unicem, 2003.

BASTOS, R. Áreas de uso comum por agricultores tradicionais de comunidades da região de Morraria, Cáceres-MT. 2004. 139f. Dissertação (Mestrado em Agricultura Tropical) - Faculdade de Agronomia e Medicina Veterinária, Universidade Federal de Mato Grosso, Cuiabá, MT, 2004.

BRITO, M. A.; COELHO, M. F. B. Os quintais agroflorestais em regiões tropicais - unidades auto-sustentáveis. Revista de Agricultura Tropical, v.1, n.1, p. 7-38. 2000.

CLEMENT, C. R. 1492 and the loss of the Amazonian crop genetic resources. I. The relation between domestication and human population decline. Economic Botany, v. 53, n. 2, p. 188-202, 1999.

COSTA, M. C da. Condicionantes de procedimentos técnicos de agricultores tradicionais de três comunidades da região de Morraria, Cáceres, MT. 2004. 139f. Dissertação (Mestrado em Agricultura Tropical) - Faculdade de Agronomia e Medicina Veterinária, Universidade Federal de Mato Grosso, Cuiabá, MT, 2004.

COTTON, C. M. Ethonotany - principles and applications. Great Britain: J Wiley \& Sons, 1996.

FOOD AND AGRICULTURE ORGANIZATION OF THE UNITED NATIONS (FAO). Household food security and community nutrition. 2004. Disponível em: <http://www. fao.org/es/ESN/nutrition/household_gardens_en.stm>. Acesso em: 5 mar. 2010.

GODOY, D. P. dos S. Estrutura e função dos quintais de unidades produtivas das comunidades Nossa Senhora da Guia e Santana, Cáceres MT. 2004. 112f. Dissertação (Mestrado em Agricultura Tropical) - Faculdade de Agronomia e Medicina Veterinária, Universidade Federal de Mato Grosso, Cuiabá, MT, 2004.

HART, R. D. Conceptos basicos sobre agroecossistema. Turrialba, Costa Rica: Centro Agronómico Tropical de Investigacion e Ensenanza - CATIE, 1985.

INSTITUTO BRASILEIRO DE GEOGRAFIA E ESTATÍSTICA (IBGE). Estudo Nacional 
de Despesa Familiar (ENDEF) - Tabelas de Composição de Alimentos. 2. ed. Rio de Janeiro, 1981. 213p.

LAKATOS, E. M. Metodologia do trabalho científico. 4. ed. São Paulo: Atlas, 1985.

MADALENO, I. Urban agriculture in Belém, Brazil. Cities, v. 17, n. 1, p. 73-77, 2000.

MENDES, R. R. Manejo e uso da vegetação nativa em unidades produtivas da Comunidade Santana, Região da Morraria, Cáceres-MT. 2005. 104f. Dissertação (Mestrado em Agricultura Tropical) - Faculdade de Agronomia e Medicina Veterinária, Universidade Federal de Mato Grosso, Cuiabá, MT, 2005.

MONTEIRO, R.; MONTEIRO S. T. Notas sobre metodologia de pesquisa. Cuiabá, MT: COOTRADE, 2000. 26p.

OLIVEIRA, R. C. de. Uso e manejo de recursos nos arredores das residências de camponeses: estudo de caso na região de Morraria. 2006. 166f. Dissertação (Mestrado em Agricultura Tropical) - Faculdade de Agronomia e Medicina Veterinária, Universidade Federal de Mato Grosso, Cuiabá, MT, 2006.

RAMOS, A. de A. Estratégias deocupação doespaço e uso de recursos naturais em unidades de produção da comunidade de Santana, Cáceres-MT. 2006. 212f. Dissertação (Mestrado em Agricultura Tropical) - Faculdade de Agronomia e Medicina Veterinária, Universidade Federal de Mato Grosso, Cuiabá, MT, 2006.

SANTOS A. M.; MITJA, D. Agricultura familiar e desenvolvimento local: os desafios para a sustentabilidade econômico-ecológica na comunidade de Palmares II, Parauapebas, PA. Interações, v. 13, n. 1, p. 39-48, jan./jun. 2012.

SEMEDO, R. J. C. G.; BARBOSA, R. I. Árvores frutíferas nos quintais urbanos de Boa Vista, Roraima, Amazônia brasileira. Acta Amazonica, v. 37, n. 4, p. 497-504, 2007.

TABOSA, F. J. S; TEIXEIRA, K. H.; SILVA, D. M. F.; MADALOZZO, C. L.; MAYORGA, M. I. O. Desenvolvimento local e capital social: uma leitura sobre os núcleos e arranjos produtivos do estado do Ceará. 2012. Disponível em: <http://www.sober.org.br/ palestra/12/05P308.pdf>. Acesso em: 5 mar. 2015.

VIEIRA, S. N. As plantas medicinais dos quintais de Mimoso. Uma abordagem através da Educação Ambiental. 2003. 35f. (Monografia de Licenciatura em Ciências Biológicas) Instituto de Biociências, Universidade Federal de Mato Grosso, Cuiabá, MT, 2003.

VIERTLER, R. B. Métodos antropológicos como ferramenta para estudos em Etnobiologia e Etnoecologia. In: AMOROZO, M .C. M.; MING, L. C.; SILVA, S. P. (Ed.). Métodos de coleta e análise de dados em Etnobiologia, Etnoecologia e disciplinas correlatas. Rio Claro: SP: UNESP/CNPq, 2002.

WINKLERPRINS, A. M. G. A. House-lot gardens in Santarém, Pará, Brazil: Linking rural with urban. Urban Ecosystems, v. 6,n. 1, p. 43-65, mar. 2002.

\section{Sobre os autores:}

Sandro Nunes Vieira: Programa de Pós-graduação em Agricultura Tropical, Universidade Federal de Mato Grosso (UFMT), Cuiabá, MT. E-mail: sandrovieira@ gmail.com

Rodrigo Aleixo Brito de Azevedo: Instituto de Desenvolvimento Rural, Universidade da Integração Internacional da Lusofonia Afro-Brasileira (UNILAB), Redenção, CE. E-mail: rodrigo_azevedo@unilab.edu.br

Maria de Fatima Barbosa Coelho: Professora no Programa de Pós-Graduação em Agricultura Tropical da Universidade Federal de Mato Grosso (UFMT), Cuiabá, MT. Áreas de pesquisa: conservação de recursos genéticos, etnobotânica e propagação de plantas nativas. E-mail: coelhomfstrela@gmail.com

Regiane Correia de Oliveira: Programa de Pós-Graduação em Agricultura Tropical da Universidade Federal de Mato Grosso (UFMT), Cuiabá, MT. E-mail: regiane@gmail.com 